\title{
Optimal couples of rearrangement invariant spaces for the Riesz potential on the bounded domain
}

Shin Min Kang ${ }^{1}$, Arif Rafiq ${ }^{2}$, Waqas Nazir ${ }^{2}$, Irshaad Ahmad ${ }^{3}$, Faisal Ali ${ }^{4}$ and Young Chel Kwun ${ }^{*}$

\section{"Correspondence:}

yckwun@dau.ac.kr

${ }^{5}$ Department of Mathematics,

Dong-A University, Pusan, 614-714,

Korea

Full list of author information is

available at the end of the article

\begin{abstract}
We prove continuity of the Riesz potential operator in optimal couples of rearrangement invariant function spaces defined in $\mathbf{R}^{n}$ with the Lebesgue measure. MSC: 46E30; 46E35

Keywords: Riesz potential operator; rearrangement invariant function spaces; real interpolation
\end{abstract}

\section{Introduction}

Let $\mathcal{M}$ be the space of all locally integrable functions $f$ on $\Omega \subset \mathbf{R}^{n}$ with the Lebesgue measure, finite almost everywhere, and let $\mathcal{M}^{+}$be the space of all non-negative locally integrable functions on $(0, \infty)$ with respect to the Lebesgue measure, finite almost everywhere. We shall also need the following two subclasses of $\mathcal{M}^{+}$. The subclass $M$ consists of those elements $g$ of $\mathcal{M}^{+}$for which there exists an $m>0$ such that $t^{m} g(t)$ is increasing. The subclass $M_{0}$ consists of those elements $g$ of $\mathcal{M}^{+}$which are decreasing.

The Riesz potential operator $R_{\Omega}^{s}, 0<s<n, n \geq 1$ is defined formally by

$$
R_{\Omega}^{s} f(x)=\int_{\Omega} f(y)|x-y|^{s-n} d y, \quad f \in \mathcal{M}^{+} ; \quad|\Omega|=1
$$

We shall consider rearrangement invariant quasi-Banach spaces $E$, continuously embedded in $L^{1}\left(\mathbf{R}^{n}\right)+L^{\infty}\left(\mathbf{R}^{n}\right)$, such that the quasi-norm $\|f\|_{E}$ in $E$ is generated by a quasi-norm $\rho_{E}$, defined on $\mathcal{M}^{+}$with values in $[0, \infty]$, in the sense that $\|f\|_{E}=\rho_{E}\left(f^{*}\right)$. In this way equivalent quasi-norms $\rho_{E}$ give the same space $E$. We suppose that $E$ is nontrivial. Here $f^{*}$ is the decreasing rearrangement of $f$, given by

$$
f^{*}(t)=\inf \left\{\lambda>0: \mu_{f}(\lambda) \leq t\right\}, \quad t>0
$$

where $\mu_{f}$ is the distribution function of $f$, defined by

$$
\mu_{f}(\lambda)=\left|\left\{x \in \mathbf{R}^{n}:|f(x)|>\lambda\right\}\right|_{n}
$$

$|\cdot|_{n}$ denoting the Lebesgue $n$-measure.

Note that $f^{*}(t)=0$, if $t>1$.

\section{Springer}

O2014 Kang et al.; licensee Springer. This is an Open Access article distributed under the terms of the Creative Commons Attribution License (http://creativecommons.org/licenses/by/2.0), which permits unrestricted use, distribution, and reproduction in any medium, provided the original work is properly cited. 
There is an equivalent quasi-norm $\rho_{p}$ that satisfies the triangle inequality $\rho_{p}^{p}\left(g_{1}+g_{2}\right) \leq$ $\rho_{p}^{p}\left(g_{1}\right)+\rho_{p}^{p}\left(g_{2}\right)$ for some $p \in(0,1)$ that depends only on the space $E$ (see [1]).

We say that the norm $\rho_{E}$ is $K$-monotone (cf. [2], p.84, and also [3], p.305) if

$$
\int_{0}^{t} g_{1}^{*}(s) d s \leq \int_{0}^{t} g_{2}^{*}(s) d s \quad \text { implies } \quad \rho_{E}\left(g_{1}^{*}\right) \leq \rho_{E}\left(g_{2}^{*}\right), \quad g_{1}, g_{2} \in \mathcal{M}^{+} .
$$

Then $\rho_{E}$ is monotone, i.e., $g_{1} \leq g_{2}$ implies $\rho_{E}\left(g_{1}\right) \leq \rho_{E}\left(g_{2}\right)$.

We use the notations $a_{1} \lesssim a_{2}$ or $a_{2} \gtrsim a_{1}$ for non-negative functions or functionals to mean that the quotient $a_{1} / a_{2}$ is bounded; also, $a_{1} \approx a_{2}$ means that $a_{1} \lesssim a_{2}$ and $a_{1} \gtrsim a_{2}$. We say that $a_{1}$ is equivalent to $a_{2}$ if $a_{1} \approx a_{2}$.

We say that the norm $\rho_{E}$ satisfies the Minkovski inequality if for the equivalent quasinorm $\rho_{p}$,

$$
\rho_{p}^{p}\left(\sum g_{j}\right) \lesssim \sum \rho_{p}^{p}\left(g_{j}\right), \quad g_{j} \in \mathcal{M}^{+}
$$

For example, if $E$ is a rearrangement invariant Banach function space as in [3], then by the Luxemburg representation theorem $\|f\|_{E}=\rho_{E}\left(f^{*}\right)$ for some norm $\rho_{E}$ satisfying (1.2) and (1.3). More general example is given by the Riesz-Fischer monotone spaces as in [3], p.305.

Recall the definition of the lower and upper Boyd indices $\alpha_{E}$ and $\beta_{E}$. Let

$$
h_{E}(u)=\sup \left\{\frac{\rho_{E}\left(g_{u}^{*}\right)}{\rho_{E}\left(g^{*}\right)}: g \in \mathcal{M}^{+}\right\}, \quad g_{u}(t):=g(t / u)
$$

be the dilation function generated by $\rho_{E}$. Then

$$
\alpha_{E}:=\sup _{0<t<1} \frac{\log h_{E}(t)}{\log t} \quad \text { and } \quad \beta_{E}:=\inf _{1<t<\infty} \frac{\log h_{E}(t)}{\log t} .
$$

If $\rho_{E}$ is monotone, then the function $h_{E}$ is submultiplicative, increasing, $h_{E}(1)=1$, $h_{E}(u) h_{E}(1 / u) \geq 1$, hence $0 \leq \alpha_{E} \leq \beta_{E}$. If $\rho_{E}$ is $K$-monotone, then by interpolation (analogously to [3], p.148), we see that $h_{E}(s) \leq \max (1, s)$. Hence in this case we have also $\beta_{E} \leq 1$.

Using the Minkovski inequality for the equivalent quasi-norm $\rho_{p}$ and monotonicity of $f^{*}$, we see that

$$
\rho_{E}\left(f^{*}\right) \approx \rho_{E}\left(f^{* *}\right) \quad \text { if } \beta_{E}<1,
$$

where $f^{* *}(t)=\frac{1}{t} \int_{0}^{t} f^{*}(s) d s$. The main goal of this paper is to prove continuity of the Riesz potential operator $R_{\Omega}^{s}: E \mapsto G$ in optimal couples of rearrangement invariant function spaces $E$ and $G$, where $\|f\|_{G}:=\rho_{G}\left(f^{*}\right)$. It is convenient to introduce the following classes of quasi-norms, where the optimality of $R_{\Omega}^{s}: E \mapsto G$ is investigated. Let $\mathcal{N}_{d}$ stand for all domain quasi-norms $\rho_{E}$, which are monotone, rearrangement invariant, satisfying Minkowski's inequality, $\rho_{E}\left(\chi_{(0,1)}\right)<\infty$ and

$$
E \hookrightarrow L^{1}(\Omega)
$$


Let $\mathcal{N}_{t}$ consist of all target quasi-norms $\rho_{G}$ that are monotone, satisfy Minkowski's inequality, $\rho_{G}\left(\chi_{(0,1)}\right)<\infty, \rho_{G}\left(\chi_{(1, \infty)} t^{s / n-1}\right)<\infty$ and

$$
G \hookrightarrow \Lambda^{\infty}\left(t^{1-s / n}\right)\left(\mathcal{R}^{n}\right)
$$

where $\chi_{(a, b)}$ is the characteristic function of the interval $(a, b), 0<a<b \leq \infty$. Note that technically it is more convenient not to require that the target quasi-norm $\rho_{G}$ is rearrangement invariant. Of course, the target space $G$ is rearrangement invariant, since $\|f\|_{G}=\rho_{G}\left(f^{*}\right)$. Finally, let $\mathcal{N}:=\mathcal{N}_{d} \times \mathcal{N}_{t}$.

Definition 1.1 (Admissible couple) We say that the couple $\left(\rho_{E}, \rho_{G}\right) \in \mathcal{N}$ is admissible for the Riesz potential if the following estimate is valid:

$$
\rho_{G}\left(\left(R_{\Omega}^{s} f\right)^{* *}\right) \lesssim \rho_{E}\left(f^{*}\right)
$$

Moreover, $\rho_{E}(E)$ is called domain quasi-norm (domain space), and $\rho_{G}(G)$ is called a target quasi-norm (target space).

For example, by Theorem 2.2 below (the sufficient part), the couple $E=\Lambda^{q}\left(t^{s / n} w\right)(\Omega)$, $G=\Lambda^{q}(v), 1 \leq q \leq \infty$, is admissible if $\beta_{E}<1$ and $v$ is related to $w$ by the Muckenhoupt condition [4]:

$$
\left(\int_{0}^{t}[v(s)]^{q} d s / s\right)^{1 / q}\left(\int_{t}^{\infty}[w(s)]^{-r} d s / s\right)^{1 / r} \lesssim 1, \quad 1 / q+1 / r=1 .
$$

Definition 1.2 (Optimal target quasi-norm) Given the domain quasi-norm $\rho_{E} \in \mathcal{N}_{d}$, the optimal target quasi-norm, denoted by $\rho_{G(E)}$, is the strongest target quasi-norm, i.e.,

$$
\rho_{G}\left(g^{*}\right) \lesssim \rho_{G(E)}\left(g^{*}\right), \quad g \in \mathcal{M}^{+},
$$

for any target quasi-norm $\rho_{G} \in \mathcal{N}_{t}$ such that the couple $\rho_{E}, \rho_{G}$ is admissible.

Definition 1.3 (Optimal domain quasi-norm) Given the target quasi-norm $\rho_{G} \in \mathcal{N}_{t}$, the optimal domain quasi-norm, denoted by $\rho_{E(G)}$, is the weakest domain quasi-norm, i.e.,

$$
\rho_{E(G)}\left(g^{*}\right) \lesssim \rho_{E}\left(g^{*}\right), \quad g \in \mathcal{M}^{+},
$$

for any domain quasi-norm $\rho_{E} \in \mathcal{N}_{d}$ such that the couple $\rho_{E}, \rho_{G}$ is admissible.

Definition 1.4 (Optimal couple) The admissible couple $\rho_{E}, \rho_{G}$ is said to be optimal if $\rho_{E}=\rho_{E(G)}$ and $\rho_{G}=\rho_{G(E)}$.

The optimal quasi-norms are uniquely determined up to equivalence, while the corresponding optimal quasi-Banach spaces are unique.

\section{Admissible couples}

Here we give a characterization of all admissible couples $\left(\rho_{E}, \rho_{G}\right) \in \mathcal{N}$. It is convenient to define the case $\beta_{E}=1$ as limiting and the case $\beta_{E}<1$ as sublimiting. 
Theorem 2.1 (General case $\left.\beta_{E} \leq 1\right)$ The couple $\left(\rho_{E}, \rho_{G}\right) \in \mathcal{N}$ is admissible if and only if

$$
\rho_{G}\left(\chi_{(0,1)} S_{1} g\right) \lesssim \rho_{E}(g), \quad g \in \mathcal{M}^{+} \text {or } g \in M_{0}
$$

where

$$
S_{1} g(t):= \begin{cases}t^{s / n-1} \int_{0}^{t} g(u) d u+\int_{t}^{1} u^{s / n} g(u) d u / u, & 0<t<1,0<s<n, n \geq 1, \\ t^{s / n-1} \int_{0}^{1} g(u) d u, & t>1,0<s<n, n \geq 1 .\end{cases}
$$

Proof First we prove

$$
\left(R_{\Omega}^{s} f\right)^{* *} \lesssim S_{1} f^{*}
$$

We are going to use real interpolation for quasi-Banach spaces. First we recall some basic definitions. Let $\left(A_{0}, A_{1}\right)$ be a couple of two quasi-Banach spaces (see $\left.[2,5]\right)$ and let

$$
K(t, f)=K\left(t, f ; A_{0}, A_{1}\right)=\inf _{f=f_{0}+f_{1}}\left\{\left\|f_{0}\right\|_{A_{0}}+t\left\|f_{1}\right\|_{A_{1}}\right\}, \quad f \in A_{0}+A_{1}
$$

be the $K$-functional of Peetre (see [2]). By definition, the $K$-interpolation space $A_{\Phi}=$ $\left(A_{0}, A_{1}\right)_{\Phi}$ has a quasi-norm

$$
\|f\|_{A_{\Phi}}=\|K(t, f)\|_{\Phi}
$$

where $\Phi$ is a quasi-normed function space with a monotone quasi-norm on $(0, \infty)$ with the Lebesgue measure and such that $\min \{1, t\} \in \Phi$. Then (see [5])

$$
A_{0} \cap A_{1} \hookrightarrow A_{\Phi} \hookrightarrow A_{0}+A_{1}
$$

where by $X \hookrightarrow Y$ we mean that $X$ is continuously embedded in $Y$. If $\|g\|_{\Phi}=\left(\int_{0}^{\infty} t^{-\theta q} \times\right.$ $\left.g^{q}(t) d t / t\right)^{1 / q}, 0<\theta<1,0<q \leq \infty$, we write $\left(A_{0}, A_{1}\right)_{\theta, q}$ instead of $\left(A_{0}, A_{1}\right)_{\Phi}$ (see [2]).

Using the Hardy-Littlewood inequality $\int_{\mathbf{R}^{n}}|f(x) g(x)| d x \leq \int_{0}^{\infty} f^{*}(t) g^{*}(t) d t$, we get the well-known mapping property

$$
R_{\Omega}^{s}: \Lambda^{1}\left(t^{s / n}\right)(\Omega) \mapsto L^{\infty}\left(\mathcal{R}^{n}\right)
$$

and by the Minkovski inequality for the norm $f^{* *}$ we get

$$
R_{\Omega}^{s}: L^{1}(\Omega) \mapsto \Lambda^{\infty}\left(t^{1-s / n}\right)\left(\mathcal{R}^{n}\right) .
$$

Hence

$$
t^{1-s / n}\left(R_{\Omega}^{s} f\right)^{* *}(t) \lesssim K\left(t^{1-s / n}, f ; L^{1}(\Omega), \Lambda^{1}\left(t^{s / n}\right)(\Omega)\right)
$$

therefore (see [2], Section 5.7)

$$
t^{1-s / n}\left(R_{\Omega}^{s} f\right)^{* *}(t) \lesssim \begin{cases}\int_{0}^{t} f^{*}(u) d u+t^{1-s / n} \int_{t}^{1} u^{s / n} f^{*}(u) d u / u, & 0<t<1 \\ \int_{0}^{1} f^{*}(u) d u, & t>1\end{cases}
$$


implies

$$
\left(R_{\Omega}^{s} f\right)^{* *}(t) \lesssim S_{1} f^{*}(t)
$$

It is clear that (1.7) follows from (2.1) and (2.3).

Now we prove that (1.7) implies (2.1). To this end we choose the test function in the form $f(x)=g\left(c|x|^{n}\right), g \in \mathcal{M}^{+}$, so that $f^{*}(t)=g^{*}(t)$ for some positive constant $c(c f$. [6]). Then

$$
R_{\Omega}^{s} f(x)=\int_{|y|<|x|} g\left(c|y|^{n}\right)|x-y|^{s-n} d y+\int_{|y|>|x|} g\left(c|y|^{n}\right)|x-y|^{s-n} d y,
$$

whence

$$
\left|R_{\Omega}^{s} f(x)\right| \gtrsim|x|^{s-n} \int_{0}^{c|x|^{n}} g(u) d u+\int_{c|x|^{n}}^{|\Omega|=1} u^{s / n-1} g(u) d u \gtrsim \chi_{(0,1)}\left(S_{1} g\right)\left(c|x|^{n}\right) .
$$

Note that $\chi_{(0,1)} S_{1} g \approx \chi_{(0,1)} Q_{1} T_{1}^{\prime} g+\chi_{(0,1)} \int_{0}^{1} g(u) d u$, where

$$
Q_{1} g:=\int_{t}^{1} g(u) d u / u, \quad t<1,
$$

and

$$
T_{1}^{\prime} g(t):= \begin{cases}t^{s / n-1} \int_{0}^{t} g(u) d u, & 0<t<1,0<s<n, n \geq 1, \\ t^{s / n-1} \int_{0}^{1} g(u) d u, & t>1,0<s<n, n \geq 1\end{cases}
$$

hence $\chi_{(0,1)} S_{1} g$ is decreasing, therefore

$$
\left|R_{\Omega}^{s} f\right|^{*}(t) \gtrsim \chi_{(0,1)} S_{1} g(t) .
$$

Thus, if (1.7) is given, then (2.4) implies (2.1).

In the sublimiting case $\beta_{E}<1$ we can simplify the condition (2.1), replacing $S_{1}$ by $T_{1}$. Here

$$
T_{1} g(t):=\left\{\begin{array}{l}
t^{s / n-1} \int_{t}^{1} u^{s / n} g(u) d u / u, \quad 0<t<1,0<s<n, n \geq 1 \\
0, \quad t>1 .
\end{array}\right.
$$

Theorem 2.2 (Sublimiting case $\beta_{E}<1$ ) The couple $\left(\rho_{E}, \rho_{G}\right) \in \mathcal{N}$ is admissible if and only if

$$
\rho_{G}\left(\chi_{(0,1)} T_{1} g\right) \lesssim \rho_{E}(g), \quad g \in M,
$$

where we recall that

$$
M:=\left\{g \in \mathcal{M}^{+} \text {and } t^{m} g(t) \text { is increasing for some } m>0\right\} .
$$


Proof Let $\rho_{E}, \rho_{G}$ be an admissible couple, then

$$
\rho_{G}\left(\chi_{(0,1)} S_{1} g\right) \lesssim \rho_{E}(g)
$$

Since $\rho_{G}\left(\chi_{(0,1)} T_{1} g\right) \lesssim \rho_{G}\left(\chi_{(0,1)} S_{1} g\right)$, it follows that $\rho_{G}\left(\chi_{(0,1)} T_{1} g\right) \lesssim \rho_{E}(g), g \in M$. Now we need to prove sufficiency of (2.6). We have

$$
\chi_{(0,1)} S_{1} g^{*} \approx \chi_{(0,1)} T_{1} g^{* *}+\chi_{(0,1)} g^{* *}(1),
$$

so

$$
\rho_{G}\left(\chi_{(0,1)} S_{1} g^{*}\right) \lesssim \rho_{G}\left(\chi_{(0,1)} T_{1} g^{* *}\right)+\rho_{G}\left(\chi_{(0,1)}\right) g^{* *}(1)
$$

implies

$$
\rho_{G}\left(\chi_{(0,1)} S_{1} g^{*}\right) \lesssim \rho_{E}\left(g^{*}\right)
$$

In the subcritical case $\alpha_{E}>s / n$ we have another simplification of (2.1).

Theorem 2.3 (Case $\left.\alpha_{E}>s / n\right)$ The couple $\left(\rho_{E}, \rho_{G}\right) \in \mathcal{N}$ is admissible if and only if

$$
\rho_{G}\left(\chi_{(0,1)} T_{1}^{\prime} g\right) \lesssim \rho_{E}(g), \quad g \in M_{0}:=\left\{g \in \mathcal{M}^{+}, g \text { is decreasing }\right\}
$$

where

$$
T_{1}^{\prime} g(t):= \begin{cases}t^{s / n-1} \int_{0}^{t} g(u) d u, & 0<t<1,0<s<n, n \geq 1, \\ t^{s / n-1} \int_{0}^{1} g(u) d u, & t>1,0<s<n, n \geq 1 .\end{cases}
$$

Proof Let $\left(\rho_{E}, \rho_{G}\right) \in \mathcal{N}$ be admissible, then

$$
\rho_{G}\left(\chi_{(0,1)} S_{1} g\right) \lesssim \rho_{E}(g), \quad g \in M_{0} .
$$

As

$$
\rho_{G}\left(\chi_{(0,1)} T_{1}^{\prime} g\right) \lesssim \rho_{G}\left(\chi_{(0,1)} S_{1} g\right)
$$

we have

$$
\rho_{G}\left(\chi_{(0,1)} T_{1}^{\prime} g\right) \lesssim \rho_{E}(g) .
$$

For the reverse, it is enough to check that (2.7) implies (2.1) for $g \in M_{0}$, or

$$
\rho_{G}\left(\chi_{(0,1)} T_{1} g\right) \lesssim \rho_{E}(g), \quad g \in M_{0} .
$$

As

$$
\chi_{(0,1)} T_{1} g \lesssim \chi_{(0,1)} T_{1}^{\prime}\left(t^{-s / n} \chi_{(0,1)} T_{1} g\right)
$$




$$
\rho_{G}\left(\chi_{(0,1)} T_{1} g\right) \lesssim \rho_{E}\left(t^{-s / n} \chi_{(0,1)} T_{1} g\right) \approx \rho_{E}\left(t^{-s / n} Q_{1}\left(t^{s / n} g\right)\right) \lesssim \rho_{E}(g)
$$

Here we use

$$
\rho_{E}\left(Q_{1}\left(t^{-s / n} g\right)\right) \lesssim \rho_{E}\left(t^{-s / n} g\right), \quad g \in M_{0}, \alpha_{E}>s / n, t<1
$$

\subsection{Optimal quasi-norms}

Here we give a characterization of the optimal domain and optimal target quasi-norms. We can define an optimal target quasi-norm by using Theorem 2.1.

Definition 2.4 (Construction of the optimal target quasi-norm) For a given domain quasi-norm $\rho_{E} \in \mathcal{N}_{d}$ we set

$$
\rho_{G_{E}}\left(\chi_{(0,1)} g\right):=\inf \left\{\rho_{E}(h): \chi_{(0,1)} g \leq \chi_{(0,1)} S_{1} h, h \in \mathcal{M}^{+}\right\}, \quad g \in \mathcal{M}^{+} .
$$

Then

$$
\rho_{G(E)}(g):=\rho_{G_{E}}\left(\chi_{(0,1)} g\right)+\sup _{t>1} t^{1-s / n} g
$$

Theorem 2.5 Let $\rho_{E} \in \mathcal{N}_{d}$ be a given domain quasi-norm. Then $\rho_{G(E)} \in \mathcal{N}_{t}$, the couple $\rho_{E}$, $\rho_{G(E)}$ is admissible and the target quasi-norm is optimal. By definition,

$$
G(E):=\left\{f \in \mathcal{M}: \lim _{t \rightarrow \infty} f^{*}(t)=0, \rho_{G(E)}\left(f^{*}\right)<\infty\right\} .
$$

Proof To see that $\rho_{G(E)}$ is a quasi-norm, we first prove (1.6), for that we first prove

$$
\sup _{0<t<1} t^{1-s / n} g^{*} \lesssim \rho_{G_{E}}\left(g^{*}\right), \quad g \in \mathcal{M}^{+}
$$

Take $g \in \mathcal{M}^{+}$and consider an arbitrary $h \in \mathcal{M}^{+}$such that, for $t<1, g^{*} \leq S_{1} h$. By the Hardy inequality $g^{*} \lesssim S_{1}\left(h^{*}\right)$. Then,

$$
t^{1-s / n} g^{*} \leq K\left(t^{1-s / n}, h ; L^{1}(\Omega), \Lambda^{1}\left(t^{s / n}\right)(\Omega)\right)
$$

Hence

$$
\sup _{0<t<1} t^{1-s / n} g^{*} \leq K\left(1, h ; L^{1}(\Omega), \Lambda^{1}\left(t^{s / n}\right)(\Omega)\right) \lesssim \rho_{E}(h)
$$

Taking the infimum over all $h$ such that $g^{*} \leq S_{1} h$, we get $(2.10)$. Hence $G_{E} \hookrightarrow \Lambda^{\infty}\left(t^{1-s / n}\right)(0$, $1)$, also $\rho_{G}(\chi(1, \infty) g)=\sup _{t>1} t^{1-s / n} g$. And these two together give (1.6). $\rho_{G(E)}$ is indeed a quasi-norm on $\mathcal{M}^{+}$. Since $\chi_{(0,1)}\left(R_{\Omega}^{s} f\right)^{*} \lesssim \chi_{(0,1)} S_{1} f^{*}$, which gives $\rho_{G_{E}}\left(\chi_{(0,1)}\left(R_{\Omega}^{s} f\right)^{*}\right) \lesssim \rho_{E}\left(f^{*}\right)$. Also

$$
\sup _{t>1} t^{1-s / n}\left(R_{\Omega}^{s} f\right)^{*} \lesssim \sup _{t>1} t^{1-s / n} S_{1} f^{*}=\int_{0}^{1} f^{*}(u) d u \lesssim \rho_{E}\left(f^{*}\right)
$$


Hence $\rho_{E}, \rho_{G(E)}$ is admissible couple. Now we are going to prove that $\rho_{G(E)}$ is optimal. For this purpose, suppose that the couple $\left(\rho_{E}, \rho_{G_{1}}\right) \in \mathcal{N}$ is admissible. Then by Theorem 2.1,

$$
\rho_{G_{1}}\left(\chi_{(0,1)} S_{1} g\right) \lesssim \rho_{E}(g), \quad g \in \mathcal{M}^{+} .
$$

Therefore if $\chi_{(0,1)} g^{*} \leq \chi_{(0,1)} S_{1} h, h \in \mathcal{M}^{+}$, then

$$
\rho_{G_{1}}\left(\chi_{(0,1)} g^{*}\right) \leq \rho_{G_{1}}\left(\chi_{(0,1)} S_{1} h\right) \lesssim \rho_{E}(h)
$$

so taking the infimum on the right-hand side, we get

$$
\rho_{G_{1}}\left(\chi_{(0,1)} g^{*}\right) \lesssim \rho_{G_{E}}\left(\chi_{(0,1)} g^{*}\right)
$$

hence $\rho_{G_{1}}\left(g^{*}\right) \lesssim \rho_{G(E)}\left(g^{*}\right)$.

In the sublimiting case $\beta_{E}<1$ we can simplify the optimal target quasi-norm.

Theorem 2.6 If $\rho_{E} \in \mathcal{N}_{d}$ be a given domain quasi-norm. Then for $g \in \mathcal{M}^{+}$,

$$
\begin{aligned}
& \rho_{G_{E}}\left(\chi_{(0,1)} g^{*}\right) \approx \rho\left(\chi_{(0,1)} g^{*}\right), \\
& \rho\left(\chi_{(0,1)} g\right):=\inf \left\{\rho_{E}(h): \chi_{(0,1)} g \leq \chi_{(0,1)} T_{1} h, h \in M\right\},
\end{aligned}
$$

i.e.,

$$
\rho_{G(E)}(g) \approx \rho\left(\chi_{(0,1)} g\right)+\sup _{t>1} t^{1-s / n} g
$$

Proof If $\chi_{(0,1)} g^{*} \leq \chi_{(0,1)} T_{1} h, h \in M$, then $\chi_{(0,1)} g^{*} \leq \chi_{(0,1)} S_{1} h$, therefore

$$
\rho_{G_{E}}\left(\chi_{(0,1)} g^{*}\right) \leq \rho_{E}(h)
$$

and taking the infimum, we get

$$
\rho_{G_{E}}\left(\chi_{(0,1)} g^{*}\right) \leq \rho\left(\chi_{(0,1)} g^{*}\right) .
$$

Now for the reverse, let $\chi_{(0,1)} g^{*} \leq \chi_{(0,1)} S_{1} h, h \in \mathcal{M}^{+}$.

Then

$$
\chi_{(0,1)} g^{*} \lesssim \chi_{(0,1)} S_{1}\left(h^{*}\right) \approx \chi_{(0,1)} T_{1}\left(h^{* *}\right)+\chi_{(0,1)} f^{* *}(1),
$$

so

$$
\chi_{(0,1)} g^{*}-\chi_{(0,1)} f^{* *}(1) \lesssim \chi_{(0,1)} T_{1}\left(h^{* *}\right),
$$

which gives, since $h^{* *} \in M$,

$$
\rho\left(\chi_{(0,1)} g^{*}-\chi_{(0,1)} f^{* *}(1)\right) \lesssim \rho_{E}\left(h^{* *}\right) \approx \rho_{E}\left(h^{*}\right) \approx \rho_{E}(h),
$$


and this implies

$$
\rho\left(\chi_{(0,1)} g^{*}\right) \lesssim \rho_{E}(h)+f^{* *}(1)
$$

which gives

$$
\rho\left(\chi_{(0,1)} g^{*}\right) \lesssim \rho_{E}(h)
$$

Taking the infimum, we get $\rho\left(\chi_{(0,1)} g^{*}\right) \lesssim \rho_{G_{E}}\left(\chi_{(0,1)} g^{*}\right)$, hence $\rho\left(\chi_{(0,1)} g^{*}\right) \approx \rho_{G_{E}}\left(\chi_{(0,1)} g^{*}\right)$.

A simplification of the optimal target quasi-norm is possible also in the subcritical case $\alpha_{E}>s / n$.

Theorem 2.7 Let $\rho_{E} \in \mathcal{N}_{d}$ be a given domain quasi-norm. Then for $g \in \mathcal{M}^{+}$,

$$
\begin{aligned}
& \rho_{G_{E}}\left(\chi_{(0,1)} g^{*}\right) \approx \rho_{1}\left(\chi_{(0,1)} g^{*}\right), \\
& \rho_{1}\left(\chi_{(0,1)} g\right):=\inf \left\{\rho_{E}(h): \chi_{(0,1)} g \leq T_{1}^{\prime} h, h \in M_{0}\right\},
\end{aligned}
$$

i.e.,

$$
\rho_{G(E)}(g) \approx \rho_{1}\left(\chi_{(0,1)} g\right)+\sup _{t>1} t^{1-s / n} g
$$

Proof If $\chi_{(0,1)} g^{*} \leq \chi_{(0,1)} T_{1}^{\prime} h, h \in M_{0}$, then

$$
\chi_{(0,1)} g^{*} \leq \chi_{(0,1)} S_{1} h .
$$

Therefore

$$
\rho_{G_{E}}\left(\chi_{(0,1)} g^{*}\right) \leq \rho_{E}(h)
$$

and taking the infimum, we get

$$
\rho_{G_{E}}\left(\chi_{(0,1)} g^{*}\right) \leq \rho_{1}\left(\chi_{(0,1)} g^{*}\right) .
$$

For the reverse, let $\chi_{(0,1)} g^{*} \leq \chi_{(0,1)} S_{1} h$. Then $\chi_{(0,1)} g^{*} \lesssim \chi_{(0,1)} T_{1}\left(h^{*}\right)+\chi_{(0,1)} T_{1}^{\prime}\left(h^{*}\right)$. As

$$
\chi_{(0,1)} T_{1} g \lesssim \chi_{(0,1)} T_{1}^{\prime}\left(t^{-s / n} \chi_{(0,1)} T_{1} g\right)
$$

we get

$$
\chi_{(0,1)} g^{*} \lesssim \chi_{(0,1)} T_{1}^{\prime}\left(h^{*}+t^{-s / n} \chi_{(0,1)} T_{1}\left(h^{*}\right)\right),
$$

whence

$$
\begin{aligned}
\rho_{1}\left(\chi_{(0,1)} g^{*}\right) & \lesssim \rho_{E}\left(t^{-s / n} \chi_{(0,1)} T_{1}\left(h^{*}\right)\right)+\rho_{E}(h) \\
& \approx \rho_{E}\left(t^{-s / n} Q_{1}\left(t^{s / n} h^{*}\right)\right)+\rho_{E}(h) \\
& \lesssim \rho_{E}(h),
\end{aligned}
$$


where we use

$$
\rho_{E}\left(Q_{1}\left(t^{-s / n} g\right)\right) \lesssim \rho_{E}\left(t^{-s / n} g\right), \quad g \in M_{0}, \alpha_{E}>s / n, t<1 .
$$

Therefore, taking the infimum we arrive at

$$
\rho_{1}\left(g^{*}\right) \lesssim \rho_{G_{E}}\left(g^{*}\right)
$$

We can construct an optimal domain quasi-norm $\rho_{E(G)}$ by Theorem 2.1 as follows.

Definition 2.8 (Construction of an optimal domain quasi-norm) For a given target quasinorm $\rho_{G} \in \mathcal{N}_{t}$, we construct an optimal domain quasi-norm $\rho_{E(G)}$ by

$$
\rho_{E(G)}(g):=\rho_{G}\left(\chi_{(0,1)} S_{1} g^{*}\right), \quad g \in \mathcal{M}^{+} .
$$

Theorem 2.9 If $\rho_{G} \in \mathcal{N}_{t}$ is a given target quasi-norm, then the domain quasi-norm $\rho_{E(G)}$ is optimal. Moreover, if $\beta_{G}<1-s / n$, then the couple $\rho_{E(G)}, \rho_{G}$ is optimal.

Proof Since $\chi_{(0,1)} S_{1} g^{*} \approx \chi_{(0,1)} T_{1} g^{* *}+\chi_{(0,1)} g^{* *}(1)$, so

$$
\rho_{E(G)}(g) \approx \rho_{G}\left(\chi_{(0,1)} T_{1} g^{* *}+\chi_{(0,1)} g^{* *}(1)\right),
$$

it follows that $\rho_{E(G)}$ is a quasi-norm. To prove the property (1.5), we notice that

$$
\begin{aligned}
\rho_{E(G)}\left(f^{*}\right) & =\rho_{G}\left(\chi_{(0,1)} S_{1} f^{*}\right) \geq \rho_{G}\left(\chi_{(0,1)}\right)\left(S f^{*}\right)(1) \\
& \gtrsim \int_{0}^{1} f^{*}(t) d t \approx\|f\|_{L^{1}(\Omega)} .
\end{aligned}
$$

The couple $\rho_{E(G)}, \rho_{G}$ is admissible since $\rho_{E(G)}(g)=\rho_{G}\left(\chi_{(0,1)} S_{1} g^{*}\right) \geq \rho_{G}\left(\chi_{(0,1)} S_{1} g\right)$. Moreover, $\rho_{E(G)}$ is optimal, since for any admissible couple $\left(\rho_{E_{1}}, \rho_{G}\right) \in \mathcal{N}$ we have $\rho_{G}\left(\chi_{(0,1)} S_{1} h\right) \lesssim$ $\rho_{E_{1}}(h), h \in \mathcal{M}^{+}$. Therefore,

$$
\rho_{E(G)}\left(g^{*}\right) \leq \rho_{E_{1}}\left(g^{*}\right) .
$$

To check that if $\beta_{G}<1-s / n$, the couple $\rho_{E(G)}, \rho_{G}$ is optimal, we need only to prove that $\rho_{G}$ is an optimal target quasi-norm, i.e., $\rho\left(g^{*}\right) \lesssim \rho_{G}\left(g^{*}\right)$, where $\rho=\rho_{G(E(G))}$ is defined by (2.11), since $\beta_{E(G)}<1$. We have $\chi_{(0,1)} g^{* *}(t)-\chi_{(0,1)} g^{* *}(1)=\chi_{(0,1)} T_{1} h$, where $h(t)=t^{-s / n}\left[g^{* *}(t)-\right.$ $\left.g^{*}(t)\right] \in M, t<1$, therefore,

$$
\rho_{G_{E(G)}}\left(\chi_{(0,1)} g^{* *}(t)-\chi_{(0,1)} g^{* *}(1)\right) \leq \rho_{E(G)}(h)=\rho_{G}\left(\chi_{(0,1)} S_{1} h^{*}\right)
$$

implies

$$
\rho_{G_{E(G)}}\left(\chi_{(0,1)} g^{* *}(t)\right) \lesssim \rho_{G}\left(\chi_{(0,1)} S_{1} h^{*}\right)+g^{* *}(1),
$$

since

$$
\chi_{(0,1)} S_{1} h^{*}=\chi_{(0,1)} t^{s / n} h^{* *}+\chi_{(0,1)} T_{1} h^{*} \lesssim \chi_{(0,1)} t^{s / n} h^{* *}+\chi_{(0,1)} T_{1} h^{* *},
$$


so

$$
\rho_{G_{E(G)}}\left(\chi_{(0,1)} g^{* *}(t)\right) \lesssim \rho_{G}\left(\chi_{(0,1)} t^{s / n} h^{* *}\right)+\rho_{G}\left(\chi_{(0,1)} T_{1} h^{* *}\right)+g^{* *}(1) .
$$

Now we define

$$
P_{1} g(t):=\frac{1}{t} \int_{0}^{t} g(u) d u, \quad t<1 .
$$

For $t<1$, since $h^{*} \lesssim Q_{1} h$, we have $h^{* *}=P_{1} h^{*} \lesssim Q_{1} P_{1} h$, therefore $T_{1} h^{* *} \lesssim T_{1} Q_{1}\left(P_{1} h\right) \lesssim$ $T_{1}\left(P_{1} h\right)$. Also $T_{1}\left(P_{1} h\right) \approx T_{1} h+t^{s / n} P_{1} h$ and $P_{1} h \leq h^{* *}$. Therefore,

$$
\begin{aligned}
\rho_{G_{E(G)}}\left(\chi_{(0,1)} g^{*}\right) & \lesssim \rho_{G}\left(\chi_{(0,1)} T_{1} h\right)+\rho_{G}\left(\chi_{(0,1)} t^{s / n} h^{* *}\right)+g^{* *}(1) \\
& \lesssim \rho_{G}\left(\chi_{(0,1)} g^{* *}\right)+\rho_{G}\left(\chi_{(0,1)} t^{s / n} h^{* *}\right)+g^{* *}(1) .
\end{aligned}
$$

For $t<1$, since $h(t) \leq t^{-s / n} g^{* *}(t)$ we have $h^{*}(t) \leq t^{-s / n} g^{* *}$, therefore using $\beta_{G}<1-s / n$, Minkowski's inequality, and monotonicity of $\rho_{G}$, we have

$$
\rho_{G}\left(\chi_{(0,1)} t^{s / n} h^{* *}\right) \lesssim \rho_{G}\left(\chi_{(0,1)} g^{* *}\right)
$$

Thus

$$
\rho_{G_{E(G)}}\left(\chi_{(0,1)} g^{*}\right) \lesssim \rho_{G}\left(\chi_{(0,1)} g^{* *}\right) \approx \rho_{G}\left(\chi_{(0,1)} g^{*}\right),
$$

hence $\rho\left(g^{*}\right) \lesssim \rho_{G}\left(g^{*}\right)$.

Example 2.10 If $G=C_{0}$ consists of all bounded continuous functions such that $f^{*}(\infty)=$ 0 and $\rho_{G}(g)=g^{*}(0)=g^{* *}(0)$, then $\alpha_{G}=\beta_{G}=0$ and $\rho_{E(G)}(g) \approx \int_{0}^{1} t^{s / n} g^{* *} d t / t$, i.e., $E=$ $\Gamma^{1}\left(t^{s / n}\right)(\Omega)$ and the couple $E, G$ is optimal.

Example 2.11 Let $G=\Lambda^{\infty}(v)$ with $\beta_{G}<1-s / n$ and let

$$
\rho_{E}(g)=\sup v(t) \int_{t}^{1} u^{s / n} g^{* *}(u) d u / u \text {. }
$$

Then, the couple $E, G$ is optimal and $\beta_{E}<1$. In particular, this is true if $v$ is slowly varying since then $\alpha_{G}=\beta_{G}=0$ and $\alpha_{E}=\beta_{E}=s / n<1$.

\subsection{Subcritical case}

Here we suppose that $s / n<\alpha_{E}$.

Theorem 2.12 (Sublimiting case $\beta_{E}<1$ ) For a given domain quasi-norm $\rho_{E} \in \mathcal{N}_{d}$ with $\rho_{E}\left(\chi_{(0,1)}(t) t^{-s / n}\right)<\infty$, we have

$$
\rho_{G_{E}}\left(\chi(0,1) g^{*}\right) \approx \rho_{E}\left(t^{-s / n} g^{*}\right) \approx \rho_{E}\left(t^{-s / n} g^{* *}\right)
$$

i.e.,

$$
\rho_{G(E)}\left(g^{*}\right) \approx \rho_{G_{E}}\left(\chi_{(0,1)} g^{*}\right)+\sup _{t>1} t^{1-s / n} g
$$

Moreover, the couple $\rho_{E}, \rho_{G(E)}$ is optimal. 
Proof If $\chi_{(0,1)} g^{*} \leq \chi_{(0,1)} T_{1}^{\prime} h, h \in M_{0}$, then for $t<1, t^{-s / n} g^{*} \leq h^{* *}$, whence

$$
\rho_{E}\left(t^{-s / n} g^{*}\right) \lesssim \rho_{E}\left(h^{* *}\right) \approx \rho_{E}\left(h^{*}\right) \approx \rho_{E}(h)
$$

Taking the infimum, we get

$$
\rho_{E}\left(t^{-s / n} g^{*}\right) \lesssim \rho_{G_{E}}\left(\chi_{(0,1)} g^{*}\right)
$$

For the reverse, we notice that $\chi_{(0,1)} T_{1}^{\prime}\left(t^{-s / n} g^{*}\right) \gtrsim \chi_{(0,1)} g^{*}=g^{*}$, hence $\rho_{G_{E}}\left(\chi_{(0,1)} g^{*}\right) \lesssim$ $\rho_{E}\left(t^{-s / n} g^{*}\right)$.

It remains to prove that the domain quasi-norm $\rho_{E}$ is also optimal. Let $\rho_{E_{1}}, \rho_{G(E)}$ be an admissible couple in $\mathcal{N}$. Then

$$
\begin{aligned}
\rho_{E_{1}}\left(g^{*}\right) & \gtrsim \rho_{G(E)}\left(\chi_{(0,1)} S_{1} g^{*}\right) \\
& =\rho_{G_{E}}\left(\chi_{(0,1)} S_{1} g^{*}\right)+\sup _{t>1} t^{1-s / n} \chi_{(0,1)} S_{1} g^{*} \\
& \approx \rho_{E}\left(t^{-s / n} \chi_{(0,1)} S_{1} g^{*}\right)+0 \\
& \gtrsim \rho_{E}\left(t^{-s / n} \chi_{(0,1)} T_{1}^{\prime} g^{*}\right) \\
& \gtrsim \rho_{E}\left(\chi_{(0,1)} g^{* *}\right) \\
& \approx \rho_{E}\left(\chi_{(0,1)} g^{*}\right) \\
& \approx \rho_{E}\left(g^{*}\right) .
\end{aligned}
$$

Now we give an example.

Example 2.13 Let

$$
E=\Lambda^{q}\left(t^{\alpha} w_{1}\right)(\Omega) \cap \Lambda^{r}\left(t^{\beta} w_{2}\right)(\Omega), \quad s / n<\alpha<\beta<1,0<q, r \leq \infty,
$$

where $w_{1}$ and $w_{2}$ are slowly varying. Then we have $\alpha_{E}=\alpha, \beta_{E}=\beta$. Now by applying the previous theorem, we get

$$
G(E)=\Lambda_{0}^{q}\left(t^{\alpha-s / n} w_{1}\right) \cap \Lambda_{0}^{r}\left(t^{\beta-s / n} w_{2}\right)
$$

and the couple $(E, G(E))$ is optimal.

In the limiting case $\beta_{E}=1$, the formula for the optimal target quasi-norm is more complicated.

Theorem 2.14 (Limiting case) Let

$$
\rho_{E}(g):=\rho_{H}\left(\chi_{(0,1)} g^{* *}\right), \quad \rho_{G_{1}}(g):=\rho_{H}\left(t^{-1} \sup _{0<u<t} u^{1-s / n} g(u)\right)
$$

where $\rho_{H}$ is a monotone quasi-norm with $\alpha_{H}=\beta_{H}=1, \rho_{H}\left(\chi_{(0,1)}\right)<\infty, \rho_{H}\left(\chi_{(1, \infty)} t^{-1}\right)<\infty$ and let

$$
\begin{aligned}
& E:=\left\{f \in \mathcal{M}: t f^{* *}(t) \rightarrow 0 \text { as } t \rightarrow 0 \text { and } \rho_{E}\left(f^{*}\right)<\infty\right\} \\
& G_{1}:=\left\{f \in \mathcal{M}: \sup _{0<u<t} u^{1-s / n} f^{* *}(u) \rightarrow 0 \text { as } t \rightarrow 0 \text { and } \rho_{G}\left(f^{*}\right)<\infty\right\} .
\end{aligned}
$$


Define

$$
\rho_{G}(g):=\rho_{G_{1}}\left(\chi_{(0,1)} g\right)+\sup _{t>1} t^{1-s / n} g
$$

Then the couple $\rho_{E}, \rho_{G}$ is optimal.

Proof Note that

$$
E \hookrightarrow L^{1}(\Omega)
$$

Indeed, $\rho_{E}\left(f^{*}\right)=\rho_{H}\left(\chi_{(0,1)} g^{* *}\right) \gtrsim f^{* *}(1)=\int_{0}^{1} f^{*}(u) d u$. Hence the above embedding follows. Consequently, $\rho_{E} \in \mathcal{N}_{d}$. On the other hand,

$$
\begin{aligned}
\rho_{G}\left(f^{*}\right) & \geq \rho_{H}\left(\chi(1, \infty) t^{-1} \sup _{0<u<t} u^{1-s / n} f^{*}(u)\right) \\
& \geq \sup _{0<u<1} u^{1-s / n} f^{*}(u) \rho_{H}\left(\chi_{(1, \infty)} t^{-1}\right) .
\end{aligned}
$$

Hence $G_{1} \hookrightarrow \Lambda^{\infty}\left(t^{1-s / n}\right)(0,1)$. This together with $\rho_{G}\left(\chi_{(1, \infty)}\right)=\sup _{t>1} t^{1-s / n} g$ gives $G \hookrightarrow$ $\Lambda^{\infty}\left(t^{1-s / n}\right)$. Then from the conditions on $G_{1}$ it follows that $\rho_{G} \in \mathcal{N}_{t}$. Also, $\alpha_{E}=\beta_{E}=1$ and $\alpha_{G}=\beta_{G}=1-s / n$. On the other hand, if $0<u<1$, then

$$
u^{1-s / n}\left(R_{\Omega}^{s} f\right)^{* *}(u) \lesssim \int_{0}^{u} f^{*}(v) d v+u^{1-s / n} \int_{u}^{1} v^{s / n-1} f^{*}(v) d v .
$$

For every $\varepsilon>0$, we can find a $\delta>0$, such that $v f^{* *}(v)<\varepsilon$ for all $0<v<\delta$. Then for $0<t<1$,

$$
\sup _{0<u<t} u^{1-s / n}\left(R_{\Omega}^{s} f\right)^{* *}(u) \lesssim \int_{0}^{t} f^{*}(v) d v+\varepsilon+t^{1-s / n} \int_{\delta}^{1} v^{s / n-1} f^{*}(v) d v .
$$

Now it is easy to check that $\lim _{t \rightarrow 0} \sup _{0<u<t} u^{1-s / n}\left(R_{\Omega}^{s} f\right)^{* *}=0$ if $f \in E$.

To prove that $R^{s}: E \rightarrow G$ we need to check that the couple $\rho_{E}, \rho_{G}$ is admissible. We write for $t<1$,

$$
T_{1}^{\prime} g(t)=T_{1}^{\prime} g^{*}(t)=t^{s / n} g^{* *}(t), \quad g \in M_{0}
$$

Then

$$
\begin{aligned}
\rho_{G}\left(\chi_{(0,1)} T_{1}^{\prime} g\right) & =\rho_{G_{1}}\left(\chi_{(0,1)} T_{1}^{\prime} g\right)+\sup _{t>1} t^{1-s / n} \chi_{(0,1)} T_{1}^{\prime} g \\
& =\rho_{H}\left(\chi_{(0,1)} t^{-1} \sup _{0<u<t} u^{1-s / n} T_{1}^{\prime} g(u)\right)+\sup _{t>1} t^{1-s / n} \chi_{(0,1)} T_{1}^{\prime} g \\
& =\rho_{H}\left(\chi_{(0,1)} g^{* *}\right) \\
& =\rho_{E}(g) .
\end{aligned}
$$

To prove that the target space is optimal, notice first that

$$
\sup _{0<u<t} u^{1-s / n} f^{* *}(u) \approx K\left(t^{1-s / n}, f ; \Lambda^{\infty}\left(t^{1-s / n}\right), L^{\infty}\right) .
$$


If $f \in G$, then by [2]

$$
\begin{aligned}
\sup _{0<u<t} u^{1-s / n} f^{* *}(u) & \approx \int_{0}^{t^{1-s / n}} h_{1}(u) d u \quad \text { (where } h_{1}, \text { is decreasing) } \\
& \approx \int_{0}^{t} h_{1}\left(v^{1-s / n}\right) v^{-s / n} d v \quad \text { (by a change of variables). }
\end{aligned}
$$

If $h(v)=h_{1}\left(v^{1-s / n}\right) v^{-s / n}$ then obviously $h \in M_{0}$ and

$$
\sup _{0<u<t} u^{1-s / n} f^{* *}(u) \approx \int_{0}^{t} h(v) d v=t h^{* *}(t)
$$

whence

$$
\rho_{E}(h) \approx \rho_{H}\left(\chi_{(0,1)} h^{* *}\right) \approx \rho_{H}\left(\chi_{(0,1)} t^{-1} \sup _{0<u<t} u^{1-s / n} f^{* *}(u)\right) \approx \rho_{G_{1}}\left(\chi_{(0,1)} f^{*}\right) .
$$

On the other hand,

$$
\sup _{0<u<t} u^{1-s / n} f^{* *}(u) \approx t h^{* *}(t)
$$

implies $t^{1-s / n} f^{*}(t) \lesssim t h^{* *}(t)$, which gives $f^{*} \lesssim t^{s / n} h^{* *}$, which implies $\chi_{(0,1)} f^{*} \lesssim \chi_{(0,1)} T_{1}^{\prime} h$, and therefore

$$
\rho_{G_{E}}\left(\chi_{(0,1)} f^{*}\right) \lesssim \rho_{E}(h) \lesssim \rho_{G_{1}}\left(\chi_{(0,1)} f^{*}\right)
$$

proving optimality of $G$. To check optimality of $E$, we notice that

$$
\begin{aligned}
\rho_{E(G)}(h) & =\rho_{G}\left(\chi_{(0,1)} S_{1} h^{*}\right) \gtrsim \rho_{G}\left(\chi_{(0,1)} T_{1} h^{* *}\right) \\
& \approx \rho_{H}\left(t^{-1} \sup _{0<u<t} u^{1-s / n} \chi_{(0,1)} T_{1} h^{* *}(u)\right) \\
& \gtrsim \rho_{H}\left(\chi_{(0,1)} h^{* *}\right) .
\end{aligned}
$$

Hence

$$
\rho_{E(G)}(h) \gtrsim \rho_{E}(h) .
$$

Example 2.15 Let $E=\Gamma_{0}^{\infty}(t w)(\Omega)$, consisting of all $f \in \Gamma^{\infty}(t w)(\Omega)$ such that $t f^{* *}(t) \rightarrow$ 0 as $t \rightarrow 0, w$ is slowly varying. Then $\beta_{E}=1$. If $G=\Gamma_{1}^{\infty}\left(t^{1-s / n} v\right) \cap \Gamma^{\infty}(t w)$, where $v(t)=$ $\sup _{u>t} w(u)$ and

$$
\Gamma_{1}^{\infty}(v):=\left\{f \in \Gamma^{\infty}(v): \sup _{0<u<t} u^{1-s / n} f^{*}(u) \rightarrow 0 \text { as } t \rightarrow 0\right\}
$$

then this couple is optimal. In particular, if $w=1$, then $E=L^{1}(\Omega)$ and $G=\Gamma_{1}^{\infty}\left(t^{1-s / n}\right)$. 


\section{Competing interests}

The authors declare that they have no competing interests.

\section{Authors' contributions}

All authors read and approved the final manuscript.

\section{Author details}

${ }^{1}$ Department of Mathematics and RINS, Gyeongsang National University, Jinju, 660-701, Korea. ${ }^{2}$ Department of Mathematics, Lahore Leads University, Lahore, 54810, Pakistan. ${ }^{3}$ Department of Mathematics, Government College University, Faisalabad, Pakistan. ${ }^{4}$ Centre for Advanced Studies in Pure and Applied Mathematic, Bahauddin Zakariya University, Multan, 54000, Pakistan. ${ }^{5}$ Department of Mathematics, Dong-A University, Pusan, 614-714, Korea.

\section{Acknowledgements}

This study was supported by research funds from Dong-A University.

Received: 15 October 2013 Accepted: 20 January 2014 Published: 10 Feb 2014

\section{References}

1. Köthe, G: Topologisch Lineare Räume. Springer, Berlin (1966)

2. Bergh, J, Löfström, J: Interpolation Spaces, an Introduction. Springer, Berlin (1976)

3. Bennett, C, Sharpley, R: Interpolation of Operators. Academic Press, Boston (1988)

4. Muckenhoupt, B: Hardy's inequality with weights. Stud. Math. 44, 31-38 (1972)

5. Brudny̌̌, YA, Krugliak, NY: Interpolation Spaces and Interpolation Functors. North-Holland, Amsterdam (1991)

6. Cianchi, A: Symmetrization and second-order Sobolev inequalities. Ann. Mat. Pura Appl. 183, $45-77$ (2004). doi:10.1007/s10231-003-0080-6

10.1186/1029-242X-2014-60

Cite this article as: Kang et al.: Optimal couples of rearrangement invariant spaces for the Riesz potential on the bounded domain. Journal of Inequalities and Applications 2014, 2014:60

\section{Submit your manuscript to a SpringerOpen ${ }^{\circ}$ journal and benefit from:}

- Convenient online submission

- Rigorous peer review

- Immediate publication on acceptance

- Open access: articles freely available online

- High visibility within the field

- Retaining the copyright to your article 